\title{
Determining the Differences in Image-resolutions of Single-particle CryoTEM Datasets Acquired with Indirect-electron and Direct-electron CMOS Cameras
}

\author{
Dalaver H. Anjum ${ }^{*}$, Mohamed A. Sobhy ${ }^{2}$, Ali R. Behzad ${ }^{1}$, Fahad Rashid ${ }^{2}$, Rachid Sougrat ${ }^{1}$, and Samir \\ M. Hamdan ${ }^{2}$ \\ ${ }^{1}$ King Abdullah University of Science \& Technology (KAUST), Core Labs, Thuwal, Saudi Arabia \\ 2. Laboratory of DNA Replication and Recombination, Biological and Environmental Sciences and \\ Engineering Division, King Abdullah University of Science and Technology (KAUST), Thuwal, Saudi \\ Arabia \\ * Corresponding author: dalaver.anjum@kaust.edu.sa
}

Single-particle cryoTEM techniques have revolutionized the field of structural biology because they can now provide 3-dimesional (3d) images of proteins and biological complexes at near atomic-resolutions [1]. Arguably speaking, this unprecedented progress is made possible only after the replacement of charged-coupled devices (CCD) cameras with complementary metal oxide semiconductor (CMOS) cameras [2]. CMOS cameras for TEMs are more attractive because they intrinsically possess higher speed for recording the images than their CCD counterparts. Second, they are available in both indirect-electron (with-Scintillator) and direct-electron (without-Scintillator) configurations for the detection of electrons. Direct-electron cameras are indeed superior to indirect cameras because the former possess close-to-ideal optical parameters i.e. quantum detective efficiency (QDE) and modular transfer function (MTF). Hence they are also suitable for recording cryoTEM images even under ultra-low electron dose $\left(\leq 1 \mathrm{e}^{-} / \AA^{2}\right)$ conditions. It is, however, desirable to determine a performance-based comparison for these cameras and that is why it was the objective of work presented in this report.

This comparison is done by applying the single-particle analysis (SPA) on recombinant Protocatechuate 3,4-dioxygenase (3,4-PCD) protein which is taken from Pseudomonas aeruginosa expressed in competent Escherichia coli strain. This protein is an oligomer of a relative molecular weight of $587 \mathrm{KDa}$ and is responsible for the biodegradation of aromatic compounds by catalyzing the essential ring-opening step. Data were acquired with a Titan $\mathrm{G}^{2}$ Krios microscope which was housing both indirect-electron and directelectron CMOS cameras of models OneView and K2, respectively. Oneview was installed at the pre-GIF location and while K2 was installed behind GIF-Quantum of model 968. The datasets were acquired with LatitudeS that was available in Gatan Microscopy Suite. All datasets received an electron dose of $\sim 50 \mathrm{e}^{-}$ $/ \AA^{2}$ per image at the object pixel size of 0.5 Á. They were then aligned and reconstructed with Relion [3]. The number of particles that went into the reconstructions of datasets in both cases were $\sim 10,000$ particles. Typical OneView and K2 acquired images of 3,4-PCD protein along with their fast-Fourier transforms (FFTs) are shown in Figure A and B, respectively. At same pixel-size, K2-acquired images had three times larger field-of-view than that of OneView-acquired images. The estimation of resolution from as-acquired images was done by locating the salient features in their FFTs. For instance, vitreous ice ring at around $3.7 \AA$ in the FFT of K2-acquired image was used as landmark for their resolution limit and whereas the extent of Thon rings in the FFTs of OneView-acquired images was taken as resolution limit of those images. It was thus found out that the K2-acquired images contained three times better resolution limit than OneView-acquired images. Resolution maps generated from OneView data and K2 data are shown in Figure (C) and (D), respectively. It can be noticed from there that the resolution-maps exist in a range unlike mere a number for the case of as-acquired images and furthermore this range was found out to be 4-4.5 $\AA$ and 11-20 $\AA$ for K2 and OneView, respectively. In summary, FFT-analysis of as-acquired images enables determining qualitative spatial-resolution only and whereas the resolution-maps generated from 
reconstructed data give an accurate and quantitative information on it. In conclusion, under similar TEMsettings, K2 camera outperforms OneView camera about four times for SPA applications.

\section{References:}

[1] Y Cheng et al., Cell 161 (2015) p. 438.

[2] D Contrato et al., Physics Procedia 37 (2012) p. 1504.

[3] SHW Scheres, Methods in Enzymology 579 (2016) p. 125.
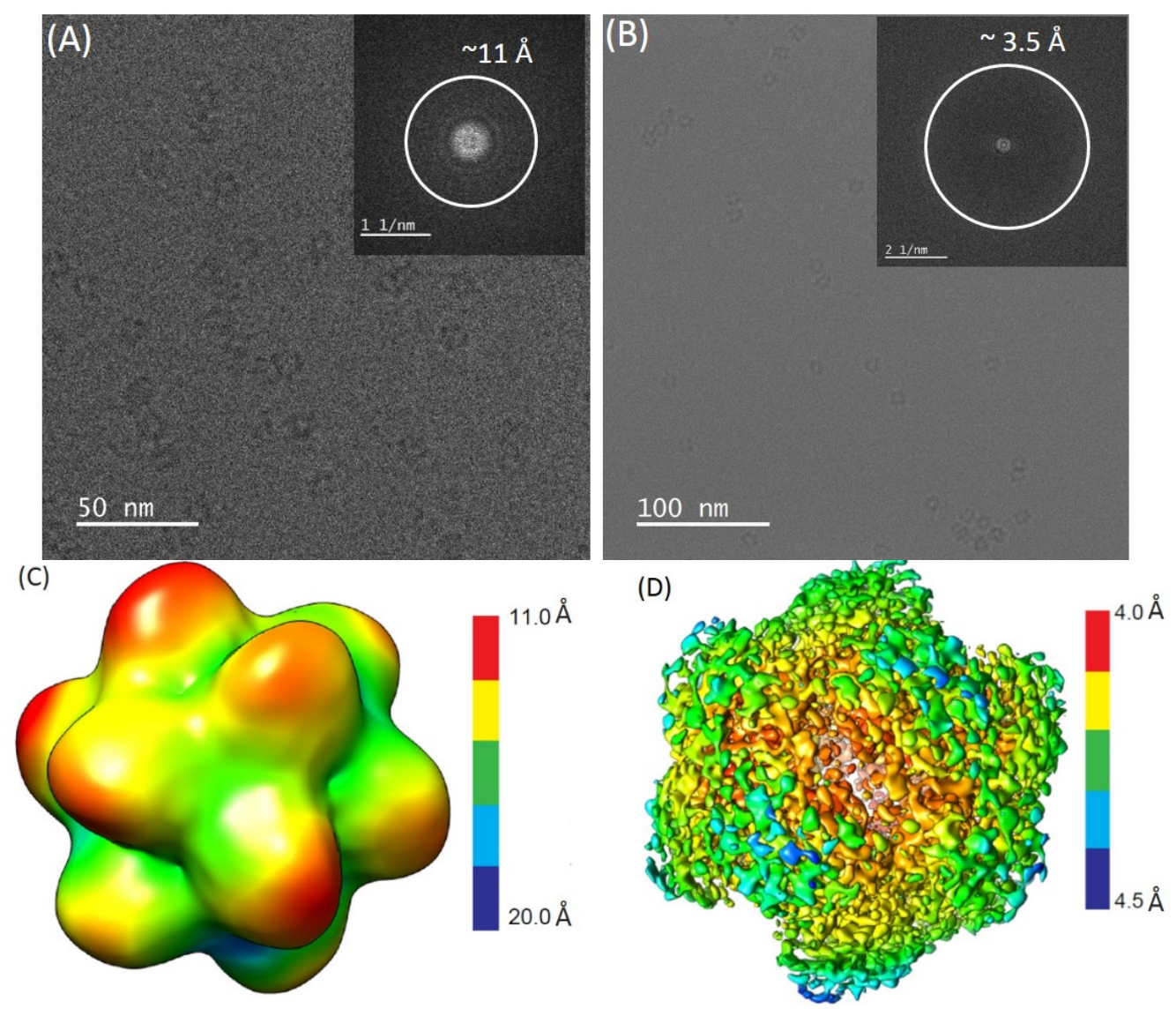

Figure 1. CryoTEM image in (A) is acquired with OneView and while the image in (B) is acquired with K2. Rings in the inserted FFTs represent their resolution. Resolution maps of 3,4-PCD proteins shown in (C) and (D) are generated from the processed OneView and K2 datasets. 